\title{
Die Eigenschaften der Bodenkörner und die Plastizität der Böden.
}

\author{
Von Albert Atterberg (Kalmar).

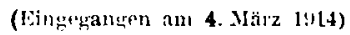

A. Einleitung.

Die Bodenarten bestehen aus Mischungen von makroskopischen, mikroskopischen und ultramikroskopischen Mineralteilen, dazu ebenfalls aus organischen Stoffen, nicht geformt (Humus) oder geformt (Torf). Die ultramikroskopischen und die feinsten mikroskopischen Teile zeigen zum großen Teil die Eigenschaften der SuspensoidKolloide. Die Humusstoffe können als Suspensoid-Aggregate aufgefaßt werden. Emulsoide scheinen in den Böden selten eine Rolle zu spielen. Wasserlösliche Kristalloide sind in einiger Menge nur in den Meeresbildungen und in den Steppenböden vorhanden. In dem Bodenwasser kommen kleinere Mengen von Kristalloiden und Kolloiden vor. Makroskopische und mikroskopische Lebewesen sind in den humosen Böden wohnhaft, werden aber als Bodenbestandteile meist nicht mitgerechnet.

$\mathrm{Da}$ die Böden an ultramikroskopischen Bestandteilen oft sehr reich sind, so mub die Bodenkundezum groben Teil innerhalb des Gebietes der Kolloidlehre fallen.

Die Böden kommen in der Natur meist mehr oder weniger wasserhaltig vor. Da sie dazu bei verschiedenen Wassergehalten sehr verschiedene Eigenschaften zeigen, so sind die Böden als gemischte, aus Bodensubstanz und Wasser bestehende Systeme aufzufassen. Wenn der Wassergehalt sinkt, dringt dazu Luft an Stelle des Wassers in dem System ein. Bei stark gelockerten Böden kann der Luftgehalt stark ansteigen.

Die Böden können sogar so viel Wasser aufnehmen, daß sie halbflüssig oder flüssig werden. Sobald sie aber flüssig werden, sind 
sie keine Böden mehr. Di e Bodenkundegehört daherzu e in e m besonderen Gebiete der Kolloidlehre, dem Gebiete der wasserarmen Systeme, wo der Wassergehalt selten so hoch steigt wie 100 Wasser : 100 trockner Bodensubstanz. Sie gehört da$z$ u einem Grenzgebiete an, dasie aufultramikroskopische Teilchen nicht beschränkt ist.

Für die quantitative Bestimmung der obengenannten Bodenbestandteile hat man die mechanische Bodenanalyse verwendet. Die jetzt gebräuchlichen Systeme der mechanischen Analyse können aber nicht såmtliche Bodenbestandteile korrekt bestimmen. Dazu wird die Bodenanalyse in verschiedenen Ländern in sehr verschiedener Weise ausgeführt. Man strebt daher jetzt dahin, neuere, verbesserte Methoden aufzustellen, die internationale Anwendung finden könnten. Vorschläge in dieser Richtung, die auf die verschiedenen Eigenschaften der Bodenkörnungen basiert sind, werden unten beschrieben.

Die Böden sind sehr verschiedener Art. Als Hauptklassen hat man meistens Tonböden, Lehmböden, Sandböden, Kalkböden, Humusund Torfböden unterschieden. Die Kalkböden werden durch hohen Gehalt an Kalziumkarbonat, die Humus- und Torfböden werden durch hohen Gehalt an organischen Stoffen gekennzeichnet. Die Tone, Lehme und Sandböden können aber nicht, wie es manchmal versucht wurde, mit Hilfe der Ergebnisse der mechanischen Bodenanalyse unterschieden werden. Sie werden durch verschiedene physikalische Eigenschaften gekennzeichnet. Die Tone sind plastische Böden, d. h. sie sind in feuchtem Zustande knetbar. Im trockenen Zustande werden sie sehr hart, und fühlen sich wegen mangelnden Sandgehaltes häufig fett an. Die Lehme sind feucht, nicht plastisch. Sie werden im trockenen Zustande weniger hart, bleiben jedoch bindig, nicht locker, und fühlen sich, wegen des Gehaltes an feinem (und grobem) Sande mager an. Die Sandböden zeigen sowohl in trockenem wie in feuchtem Zustande lockere Konsistenz.

Um diese Böden richtig klassifizieren zu können, muß man daher sowohl ihre Plast i $z$ it ät, wie ihre höhere oder niedrigere Ko nsiste $n z$ sowohl in trockenem, wie in feuchtem Zustande bestimmen können. Die Plastizitäts- und die Konsistenzverhältnisse sind aber von der Wissenschaft bisher gar zu wenig studiert worden.

Man hat für die Böden mehrere Konsistenz-Zustände zu unterscheiden. Erstens die festen kompakten Zustandsformen, bei denen die Bodenporen entweder nur luftgefüllt sind, oder sowohl Luft, wie Wasser 
enthalten. Ferner die weicheren Zustandsformen, bei denen das Wasser nicht nur die Bodenporen füllt, sondern die Bodenteilchen mehr oder weniger voneinander schiebt, dadurch das Bodenvolumen vergrössernd. Weiter die entsprechenden gelockerten Zustandsformen, wo die Bodenteilchen Aggregate bilden, die von größeren Luftkanälen getrennt sind. Die gelockerten Zustandsformen spielen in der Landwirtschaft eine große Rolle, werden aber hier nicht weiter berücksichtigt.

Diese verschiedenen Zustandsformen werden von mir Konsistenzformen genannt. Ich habe die Begriffe Konsistenzformen, Konsistenzgrenzen, Konsistenzgrade und Konsistenzkurven aufgestellt, und bin somit im Begriff für die Systeme Boden + Wasser eine be sondere Konsistenzlehre auszuarbeiten. Ein wichtiger Teil dieser Konsistenzlehre ist die Plastizitätslehre. Diese Konsistenzlehre kann sicherlich auch bei anderen Systemen Anwendung finden.

Meine Untersuchungen auf dem Gebiete der Bodenkunde haben bisher behandelt:

1. Die Eigenschaften der Bodenkörner $\left.{ }^{1}\right)$.

2. Die plastische Konsistenzform ${ }^{2}$ ).

3. Die verschiedenen Konsistenzgrade der Böden ${ }^{3}$ ).

4. Die Ursachen der Plastizität $\left.{ }^{4}\right)$.

5. Die Konsistenzkurven ${ }^{5}$.

6. Auf die Konsistenzverhältnisse und auf die Ziffern der mechanischen Bodenanalyse gegründete Klassifikationsversuche ${ }^{6}$ ).

\section{B. Die Eigenschaften und die Einteilung der Bodenkörner.}

Die mechanische Bodenanalyse ist bisher nur für humusfreie und für humusarme Böden ausgebildet. Man besitzt dazu bisher Methoden nur für das Trennen der makroskopischen und der mikroskopischen Mineralteilchen verschiedener Größe, nicht für das Trennen der ultramikroskopischen. Da diese Methoden aber das Grenzgebiet zwischen

1) Studier $\mathrm{i}$ jordanalysen, K. Landtbr. Akad. Tidskrift 1903, und Studien auf dem Gebiete der Bodenkunde, Versuchsstationen 69 (1908).

2) Die Plastizităt der Tone, Int. Mitteil. f. Bodenkunde, 1, 4 (1911).

3) Die Konsistenz und die Bindigkeit der Böden, Int. Mitteil. f. Bodenkunde 2, 149-189 (1912).

4) Die Plastizität und Bindigkeit liefernden Bestandteile der Tone. Int. Mitteil. f. Bodenk. 3, 291-330 (1913).

5) Noch nicht veröffentlicht.

6) Die mechanische Bodenanalyse und die Klassifikation der Mineralböden Schwedens. Int. Mitt. f. Bodenk. 2, 312-342 (1912). 
den mikroskopischen und den ultramikroskopischen Teilchen behandeln, so müssen sie für die Kolloidlehre ein bedeutendes Interesse bieten können, Bei den Analysen werdẹn bestimmt die Körnergruppen: Kies, Sand, Schluff und Ton (Gravier, Sable, Limon et Argile; Gravel, Sand, Silt and Clay). Diese Gruppen werden weiter eingeteilt.

Man hat sich aber nicht einigen können, wo die Grenzen zwischen Kies und Sand, zwischen Sand und Schluff und zwischen Staub und Ton zu ziehen sind. Die Grenze zwischen Kies und Sand wurde bald bei $0,4,0,9$ oder $1,0 \mathrm{~mm}$, bald bei $2,03,0$ oder $5,0 \mathrm{~mm}$ Körnerdurchmesser gezogen. Die Grenze zwischen Sand und Schluff (Staub) wurde bald bei $0,25,0,18$ oder 0,10 , bald bei $0,06,0,05,0,03$ oder $0,02 \mathrm{~mm}$ gezogen.

Die jetzt gebräuchlichsten Einteilungen der Bodenkörner sind die folgenden :

Die deutsche $e^{1}$.

Die englische ${ }^{2}$ ).

Grand, Körner, größer als $2 \mathrm{~mm}$ Fine gravel $3-1 \mathrm{~mm}$

Sand $\left\{\begin{array}{lrl}\text { sehr grob } & 2-1 \\ \text { grob } & 1-0,5 & \\ \text { mittelkörnig } & 0,5-0,2 & " \\ \text { fein } & 0,2-0,1 & \\ \text { sehr fein } & 0,1-0,05 & \end{array}\right.$ Sand $\left\{\begin{array}{lr}\text { coarse } & 1-0,3(?) \quad, \\ \text { fine } & 0,3-0,037(?)\end{array}\right.$

Silt $\left\{\begin{array}{cc}. . & 0,037-0,012 \\ \text { fine } & 0,012-0,0013\end{array}\right.$ "

Clay

$0,0013-0,00$,

Feinste tonhaltige Teile $<0,01$ "

Die amerikanis che ${ }^{3}$ ).

Fine gravel

Sand $\left\{\begin{array}{lr}\text { coarse } & 1-0,5 \quad \\ \text { medium } & 0,5-0,25 " \\ \text { fine } & 0,25-0,10 " \\ \text { very fine } & 0,10-0,05 "\end{array}\right.$

Silt

$0,05-0,005$

Clay

$$
0,005-0,000
$$

Die russische ${ }^{4}$ ).

\begin{tabular}{|c|c|c|}
\hline & $3-1$ & \\
\hline Grober Sand & $1-0,5$ & \\
\hline & $0,5-0,25$ & \\
\hline Feinar Sand & $0,25-0,05$ & \\
\hline remer sand & $0,05-0,01$ & \\
\hline Staub & $0,01-0,005$ & \\
\hline & $0,005-0,0015$ & \\
\hline Schlamm & $<0,0015$ & \\
\hline
\end{tabular}

Die französische hat keine Grenzziffern.

1) F. W an s chaff e, Wissenschaftliche Bodenuntersuchung, 3. Aufl. 1814, 48.

2) E. J. Russe1, Soil conditions and plant growth, 1912, S. 152-153; deutsche Ausgabe: Boden und Pflanze, herausgeg. von H. Brehm (Dresden 1914).

$\left.{ }^{3}\right)$ G. N. Coffey, A Study of the soils of the United States 1913, 28.

4) P. Kossowitsch, Die Schwarzerde, Int. Mitteil. f. Bodenk. 1912, 301. 
Finden sich von der Natur gezogene Grenzen in den Eigenschaften der Bodenkörner?

Die Ursache dafür, daß bei diesen Einteilungen die Grenzen so stark verschieden gezogen werden, liegt natürlich daran, daß keine von der Natur gezogene Grenzen bekannt waren. Solche natürliche Grenzen müssen sich doch vorfinden. Es ist ja wohl bekannt, daß gröbere Sande trocken sind und dann schlechte Sandböden bilden, die nicht als Kulturböden, sondern nur als Waldböden passen. Feinere Sande sind dagegen gut wasserhaltend und liefern dann bei guter Kultur die guten Sandböden. Eine Grenze zwischen den groben, trocknen, schlechten Sanden und den feinen, gut wasserbehaltenden Sanden muß sich dann wenigstens annähernd feststellen lassen.

Wenn derartige von der Natur gezogene Grenzen in den Eigenschaften von den gröberen und feineren Bodenbestandteilen bekannt wären, so würden sich die Bodenbestandteile in Glieder von bestimmten Eigenschaften einteilen lassen. Eine solche Einteilung wäre eine natürliche Einteilung, und dieselbe würde wohl Aussicht haben, internationale Geltung zu erlangen.

Um solche Grenzen aufzusuchen habe ich schon im Jahre 1902 das Studium der Eigenschaften der verschiedenen Bodenkörnungen vorgenommen.

\section{Das Untersuchungsmaterial.}

Für diese Untersuchung mußte erst ein Untersuchungsmaterial, allerlei Körnergrößen von $5 \mathrm{~mm}$ hinauf bis zu wenigstens $0,001 \mathrm{~mm}$ hinab, in genügender Reinheit hergestellt werden.

Als Ausgangsmaterial wurde benutzt: Kies, grober Sand, mittelfeiner und toniger Sand, alles Glazialbildungen aus der Kalmargegend, dazu Fließlehm (mikroskopischer Sand oder Schluff) aus Nordschweden. Dieses Material wurde zur Reinigung von Ton- und Humusaggregaten erst mit Salzsäure gekocht und dann mit schwach erwärmter Natronlauge behandelt. Die etwas gröberen Sande wurden dann durch passende, makroskopisch und mikroskopisch kontrollierte Siebe in Abteilungen verschiedener Körnergröße eingeteilt. Die etwas feineren Sande wurden vermittels des Schlämmapparates Ko pecky's $\left.{ }^{1}\right)$ - der einzige der gewöhnlichen Schlämmapparate, welcher mit größeren Bodenmengen $\mathrm{zu}$ arbeiten gestattet - in Gruppen eingeteilt. Dabei wurde jedesmal nur ein Schlämmzylinder benutzt und die Schnelligkeit

1) J. Ko pecky, Die Bodenuntersuchung (Prag 1901). 
des Wasserstromes so eingestellt, daß die gewünschten Körnergrößen erreicht wurden. $\mathrm{Da}$ bei diesem Schlämmen Wasserleitungswasser benutzt wurde, mußten die fertigen Schlämmprodukte durch Salzsäure und Natronlauge wieder gereinigt werden.

Die feinsten und die mikroskopischen Sande wurden ohne besondere Apparate in weiten niedrigen Glaszylindern dem Schlämmen unterworfen. Dabei wurde so verfahren, das man die feinen und die mikroskopischen Sandarten mit destilliertem Wasser anrührte, das Wasser eine gewisse Zeit zum Absetzen der gröberen Teilchen in der Ruhe ließ, und dann das trübe Wasser durch Abhebern von dem Bodensatze trennte. Der Bodensatz wurde dann wiederum mit destilliertem Wasser angerührt, die Mischung wurde wieder dieselbe Zeit in Ruhe gelassen und das Wasser wurde dann wieder abgehebert. So wurde weiter fortgefahren, bis das Wasser schließlich ganz klar abflob. - Später wurde das Schlämmen in ganz derselben Art wieder erneuert, jetzt aber unter Verwendung kürzerer Absetzzeiten. In solcher Weise konnten eine Reihe Körnergruppen verschiedener Körnergrößen von $0,2 \mathrm{~mm}$ hinauf bis $0,001 \mathrm{~mm}$ hinab in reinem Zustande dargestellt werden.

Die zur Gewinnung bestimmter Teilchengrößen nötigen Absetzzeiten mußten durch eine besondere Untersuchung festgestellt werden. Es wurde gefunden, dab bei einer

\begin{tabular}{|c|c|c|}
\hline $\begin{array}{l}\text { Absetzzeit } \\
\text { von }\end{array}$ & $\begin{array}{l}\text { und bei Wasserhöhen } \\
\text { von }\end{array}$ & $\begin{array}{l}\text { wurden abgeschlämmt } \\
\text { Teilchen feiner als }\end{array}$ \\
\hline 8 Stunden & $10 \mathrm{~cm}$ & $0,002 \mathrm{~mm}$ \\
\hline 2 Stunden & 10 & 0,004 \\
\hline 30 Minuten & 10 & 0,009 \\
\hline $71 / 2$ Minuten & 10 & 0,02 \\
\hline 1 Minute 56 Sekunden & 10 & 0,04 \\
\hline 25 Sekunden & 10 & 0,09 \\
\hline 15 Sekunden & $30 \ldots$ & 0,2 \\
\hline
\end{tabular}

Nach diesen Vorschriften wurden die folgenden Bodenkörnungen gewonnen :

\begin{tabular}{|c|c|c|}
\hline $5-2 \mathrm{~mm}$ & $0,2-0,09 \mathrm{~mm}$ & $0,02-0,009 \mathrm{~mm}$ \\
\hline $2-1$ & $0,09-0,04$ & $0,009-0,004$ \\
\hline $1-0,5$ & $0,04-0,02$ & $0,004-0,002$ \\
\hline
\end{tabular}


Zum Darstellen einer noch feineren Teilchengröße, von 0,002 bis $0,001 \mathrm{~mm}$ wurde ein systematisches Schlämmen so angeordnet, daß das schlämmreiche Wasser 30 Stunden nach jedem Aufschlämmen in Ruhe gelassen wurde, da nach Berechnung alle Teilchen gröber als 0,001 sich zu Boden setzen müßten, und die noch feineren Teilchen abgeschlämmt werden könnten.

Die rein dargestellten Körnungen.

Sämtliche Schlämmprodukte zeigten sich bei mikroskopischer Untersuchung frei von tonigen und humosen Aggregaten. Die Farbe der gröberen Körnungen war hellrot wegen des hohen Orthoklasgehaltes (25 Proz.). Die Farbe der Körnung 0,2-- 0,09 mm war nur rötlich und die noch feineren Körnungen waren farblos, obschon der OrthoklasGehalt fortwährend hoch war. Nur die aus dem Fließlehme dargestellten Körnungen zeigten einen Stich in Grau, wegen eines Gehaltes an Hornblende und Magnetitkörnern.

Die Körnungen gröber als 0,04 mm waren wirkliche Sande. Die Körnung 0,04-0,02 zeigte sich für die Lupe ebenfalls als wirklicher Sand. Die feineren Körnungen waren mikroskopische Sande ("Staub", "Schluff"). Nur bei den feinsten Körnungen zeigte sich bei mikroskopischer Untersuchung ein gewisser Gehalt an Kaliglimmer.

Der Analyse nach bestanden die Körnungen hauptsächlich aus etwa gleichen Mengen Kalifeldspat, Natronfeldspat und Quarz mit kleinen Mengen Kalkfeldspat und in den feineren Körnungen dazu ein wenig von Magnesia- und Eisensilikaten nebst Kaliglimmer.

Bei dem Trocknen der fertiggeschlämmten Körnungen bildeten die Körner gröber als 0,02 mm lose Massen. Die Körnungen feiner als $0,02 \mathrm{~mm}$ bildeten aber Kuchen, die erst bei Berührung oder Druck zu Pulver zerfielen. Eine Adhäsion oder Kohäsion zwischen den kleinen Körnern machte sich somit schon hier bemerkbar. Bei fallenden Körnergrößen stieg die Kohäsion immer mehr.

Wenn man die Körner, gröber als $0,02 \mathrm{~mm}$, aus einem Gefäß in das andere schüttete, so flossen die Körner jedes für sich. Körner feiner als $0,02 \mathrm{~mm}$ flieben nicht so, sondern es haften die Körner etwas zusammen, lose Aggregate bildend.

Die Hygroskopizität der Sandkörnungen.

Um die Hygroskopizität der Körnungen zu untersuchen ${ }^{1}$ ), wurden Proben derselben erst in offenen Nickelschalen zwei Wochen bei 100 ,

1) Die Vorschriften $M$ it s ch e $l$ l ch 's für die Hygroskopizitätsuntersuchungen waren im Jahre 1902 noch nicht veröffentlicht. 
dann zwei Tage über Wasser gestellt bei $16^{\circ}$ und dann wieder neun Tage über Wasser gestellt. Folgende Feuchtigkeitsprozente wurden gefunden :

\begin{tabular}{|c|c|c|c|c|}
\hline Die Körnun & In & $\begin{array}{l}\text { Zimmerluft } \\
\text { bei } 10^{\circ}\end{array}$ & \multicolumn{2}{|c|}{ über Wasser bei $16^{\circ}$} \\
\hline $0,04-0,02$ & $\mathrm{~mm}$ & 0,00 & 0,10 & 0,14 \\
\hline $0,02-0,009$ & " & 0,03 & 0,15 & 0,23 \\
\hline $0,009-0,004$ & $\eta$ & 0,05 & 0,17 & 0,25 \\
\hline $0,004-0,002$ & $"$ & 0,07 & 0,26 & 0,37 \\
\hline $0,002-0,001$ & , & 0,15 & 0,84 & 1,01 \\
\hline
\end{tabular}

Die Körnungen gröber als $0.02 \mathrm{~mm}$ sind somit bei Zimmerluft gar nicht hygroskopisch.

Die Porenvolumina.

In dem System Boden + Wasser + Luft sind es die zwischen den Bodenkörnern befindlichen Poren, die bald durch Wasser, bald durch Luft gefüllt sind. Die Größe der Porenvolumina hat also für die Bodenarten große Bedeutung. Ich untersuchte die Porenvolumina meiner Sandkörnungen in der folgenden Weise. Ein graduierter Glaszylinder mit Stopfen wurde erst gewogen, dann zu $2 / 3$ mit Sand gefüllt und wieder gewogen. Wasser wurde im Ueberschuß zugefügt. Durch fleißiges Rollen des Zylinders in verschiedenen Richtungen konnten sämtliche dem Sande anhaftenden Luftblasen entfernt werden. Die feinen Körnungen wurden zur völligen Entfernung der Luft mit Wasser gekocht und dann erst in den Zylinder eingefüllt.

\begin{tabular}{|c|c|c|c|c|}
\hline Sand von & & $\begin{array}{l}\text { Litergewicht des in } \\
\text { Wasser zusammen- } \\
\text { geschüttelten Sandes } \\
\mathrm{g}\end{array}$ & $\begin{array}{c}\text { Porenvolumen } \\
\text { des Sandes } \\
\% \\
\end{array}$ & $\begin{array}{c}\text { Daraus } \\
\text { berechnetes } \\
\text { Spez. Gewicht } \\
\mathrm{g} \\
\end{array}$ \\
\hline $5-2$ & $\mathrm{~mm}$ & 1,584 & 40,1 & 2,64 \\
\hline $2-1$ & $n$ & 1,577 & 40,4 & 2,66 \\
\hline $1-0,5$ & " & 1,540 & 41,8 & 2,65 \\
\hline $0,5-0,2$ & " & 1,580 & 40,5 & 2,65 \\
\hline $0,2-0,09$ & $"$ & 1,591 & 40,4 & 2,66 \\
\hline $0,09-0,04$ & ” & 1,583 & 41,0 & 2,68 \\
\hline $0,04-0,02$ & $n$ & 1,596 & 41,0 & 2,67 \\
\hline $0,02-0,009$ & ” & 1,560 & 42,3 & 3,06 \\
\hline $0,009-0,004$ & $"$ & 1,555 & 42,7 & 2,85 \\
\hline
\end{tabular}


Die Körner wurden dann durch fleißiges Klopfen des Zylinders möglichst stark zusammengeschüttelt. Erst wenn das Sandvolumen, auf der Graduierung abgelesen, sich konstant zeigte, wurde das Klopfen beendigt. Dann wurde der Wasserüberschuß entfernt. Das Sandvolumen wurde abgelesen und das Gewicht des so gefüllten Zylinders bestimmt. Der Versuch wurde mit neuen Körnermengen mehrmals wiederholt und die Mittelzahl der Resultate berechnet. So wurden folgende Ziffern gewonnen.

Die Porenvolumina waren daher bei diesen Sandkörnungen nur wenig wechselnd. Das Litergewicht wechselte nur zwischen 1,56 und 1,596. Das spezifische Gewicht war fast konstant, nur bei den zwei letzten Proben höher, was aus dem Gehalte dieser Körnungen an Hornblende und Magnetit zu erklären ist.

Obige Ziffern sind nicht allgemein gültig. Sande anderer Herkunft können andere Porenvolumina zeigen. Französischer Tertiärsand von $0,35-0,18 \mathrm{~mm}$, nur aus Quarz bestehend, und alluvialer Ufersand aus Oeland von 0,25 -- 0,18 Körnerdurchmesser zeigten ganz andere Ziffern.

Französischer Tertiärsand litergewicht Porenvolumen Spez. Gewiclit Sand aus Oeland

$\begin{array}{lll}1,708 & 35,9 & 2,67 \\ 1,597 & 38,8 & 2,61\end{array}$

Der Unterschied wird dadurch erklärt, daß die Körner des Tertiärsandes fast kugelig waren und die Körner des Oeland-Sandes stark abgerundet waren. Meine aus Glazialsand dargestellten Körnungen zeigten dagegen hauptsächlich stark eckige Körner.

Beim Mischen von gleichen Volumina allerlei Sandkörnungen von $1 \mathrm{~mm}$ bis $0,01 \mathrm{~mm}$ sank das Porenvolumen der Mischung bis zu 30,3 hinab. Noch niedrigere Porenvolumina, bis zu 23,0, kommen bei Moränenböden oft vor.

Die Kapillarität der Sandkörnungen, d. h. die kapillare Steighöhe des Wassers in den Sanden.

Für die Bestimmung der Kapillarität der Sandkörnungen wurden eine Anzahl Glasröhren unten ausgezogen und die ausgezogenen Spitzen mit Baumwolle verschlossen. Der Sand wurde dann eingefüllt und stark zusammengeklopft. Bei den feineren Sanden wurden die Füllungen mit Hilfe eines Glasstabes stark zusammengepackt. Um hohe Säulen zu bekommen, wurden bisweilen mehrere Glasröhren durch Gummischläuche verbunden. 
Die Röhren wurden in Wasser so tief gestellt, daß die Oberfläche des Wassers sich über die Baumwolle erhöhte. Die Steighöhe des Wassers wurde täglich notiert.

Beim Eindringen des Wassers in den Röhren setzte sich bei den feineren Sanden der benetzte Teil und es entstanden dann größere Luftblasen in den Säulen. Wenn diese Blasen nur auf der einen Seite des Rohres sich zeigten, schienen dieselben auf die Resultate keinen Einfluß zu haben.

Die Versuche ergaben die folgenden Steighöhen, in $\mathrm{mm}$ berechnet.

Maximale Steighöhe

Körnung 5,0-2,0 $\mathrm{mm} \quad 25 \mathrm{~mm}$ Die Kapillaren nicht gefüllt

$$
\begin{aligned}
& \text { " } 2,0-1,0,65, \text { in } 3 \text { Tagen erreicht } \\
& \text { " } 1,0-0,5,131 \text {, in } 4 \text { Tagen " } \\
& \text { " } 0,5-0,2 \text {, } 246 \text {, in } 7 \text { Tagen , } \\
& \text { " } 0,2-0,09,428 \text { " in } 7 \text { Tagen " } \\
& \text { " } 0,09-0,04,1055 \text { " in } 72 \text { Tagen " } \\
& \text { " } 0,04-0,02,2000 \text { (?) n in } 53 \text { Tagen }=1860 \mathrm{~mm} \\
& \text { " } 0,02-0,009, \quad \text { " in } 30 \text { Tagen }=2447 \mathrm{~mm}
\end{aligned}
$$

Die Steighöhen in 24 und 48 Stunden sind von besonderem Interesse.

\begin{tabular}{c|c|c}
\hline \hline $\begin{array}{c}\text { Körnung des Sandes } \\
\mathrm{mm}\end{array}$ & $\begin{array}{c}\text { Steighöhe } \\
\text { in 24 Stunden } \\
\mathrm{mm}\end{array}$ & $\begin{array}{c}\text { Steighöhe in den } \\
\text { folgenden 24 Stunden } \\
\mathrm{mm}\end{array}$ \\
\hline $5,0-2,0$ & 22 & 2 \\
$2,0-1,0$ & 59 & 6 \\
$1,0-0,5$ & 115 & 8 \\
$0,5-0,2$ & 214 & 16 \\
$0,2-0,09$ & 376 & 20 \\
$0,09-0,04$ & 530 & 44 \\
$0,04-0,02$ & 1153 & 207 \\
$0,02-0,009$ & 485 & 437 \\
$0,009-0,004$ & 285 & $?$ \\
$0,004-0,002$ & 143 & $?$ \\
$0,002-0,001$ & 55 & schwierig abzulesen.
\end{tabular}

Die Steighöhe in 24 resp. 48 Stunden hat für die Pflanzen große Bedeutung. In trockenen Perioden ist es der Untergrund, welcher die Pflanzen mit Wasser versorgen soll. Die Verdunstung des Wassers 
von der Bodenfläche und von den Pflanzen ist in den Tagesstunden am stärksten. Während der Nacht muß der Untergrund neue Wassermengen der Bodenoberfläche und den Pflanzen zuführen. Bei guter Drainierung ist dann derjenige Untergrund, der die gröBten Wassermengen heben kann, der vorteilhafteste. - Die Sandkörnung 0,04 bis $0,02 \mathrm{~mm} \mathrm{muB}$ voraussichtlich im allgemeinen den besten Untergrund bilden. Die gröberen Sande erfordern eine höhere Lage des Grundwassers. Bei den Sanden unter $0,02 \mathrm{~mm}$ hat aber die Lage des Grundwassers kaum eine Bedeutung mehr.

Die Körnung 0,002-0,001 $\mathrm{mm}$ hebt das Wasser schon in den ersten 24 Stunden nur $55 \mathrm{~mm}$. So feiner Sand muß darum einen weniger guten Untergrund bilden, und er ist in den Eigenschaften den schweren Tonen nahestehend.

\section{Das Verhalten der Sandkörnungen $z$ um Niederschlagswasser (beim Begießen).}

Bei Untersuchungen über die kapillaren Steighöhen des Wassers in den Sandarten sind es drei Kräfte, die das Resultat bewirken: 1. Die Adhäsion und die daraus entstehenden Menisken, welche das Wasser heben; 2. die Schwere, die dieser Hebung entgegenwirkt; 3. die Reibung in den kapillaren Poren, welche die Bewegung des Wassers verlangsamt.

Bei dem Begießen von oben wirken dagegen im ersten Augenblicke die Schwerkraft und die Adhäsion in gleicher Richtung. Das Wassser dringt schnell in den Sand hinein und wird dabei nur durch die Reibung mehr oder weniger behindert. Dann aber kommen die oberen Menisken der Wassersäulen zur Wirkung und streben das fernere Niedersinken des Wassers zu verhindern. Das Resultat wird verschieden nach der Körnung des Sandes und nach den angewandten Wassermengen.

Gröbere Sande können nur wenig Wasser binden. Bei stärkerer Wasserzufuhr drückt die Schwere das Wasser rasch durch den Sand. Etwas feinere Sande können mehr und feinere Sande bedeutende Mengen Wasser zurückhalten.

Die gröberen Sande werden deshalb wasserlassend, die feineren Sande dagegen wasserhaltend genannt. Die gröberen Sandkörnungen bilden die schlechten und die feineren die guten Sandböden in landwirtschaftlichem Sinne. Die Frage, wo die Grenze zwischen den wasserlassenden trockenen und den gut wasserbehaltenden Sanden zu ziehen ist, hat darum große praktische Bedeutung. Haupt- 
sächlich, um diese Grenze zu bestimmen, habe ich die folgenden Versuche angestellt.

Graduierte und von mir genau kalibrierte Glasröhren, unten zu einem kurzen Rohr ausgezogen und daselbst mit Baumwolle geschlossen, wurden mit Sand gefüllt. Die gröberen Sande wurden stark zusammengeschüttelt. Die feineren Sande wurden bei der Füllung der Röhren stark zusammengepackt. Die Röhren wurden dann mit genau abgemessenen Wassermengen begossen, welche so berechnet waren, daß dieselben bestimmten Niederschlagshöhen entsprachen. Sämtliche Röhren wurden dann mit Baumwolle geschlossen, um die Verdunstung des Wassers zu hindern. Die Tiefe, wohin das Wasser jedesmal drang, wurde in Millimetern bezeichnet. Die Resultate waren die folgenden:

Sand von $5-2 \mathrm{~mm}$ (Kapillarität $25 \mathrm{~mm}$ ). Schon eine Wassermenge von $2,5 \mathrm{~mm}$ Höhe sank hier nach 6 Minuten tiefer als die Kapillaritätsgrenze $(25 \mathrm{~mm})$. Nach 24 Stunden war der Wassergehalt des Sandes nur 4,8 Vol.-Proz. 5,0 mm Wasser flob rasch durch den Sand herab. - Dieser Sand wird daher von dem Niederschlagswasser nur befeuchtet, kann aber keinen nennenswerten Wasservorrat zurückhalten.

Sand von 2-1 $\mathrm{mm}$ (Kapillarität $65 \mathrm{~mm}$ ). Dieser Sand kann $5,0 \mathrm{~mm}$ Wasser oberhalb der Kapillaritätsgrenze $(65 \mathrm{~mm})$ binden. Der Wassergehalt steigt dabei zu 9,9 Vol.-Proz. Größere Mengen Wasser sinken aber weit unterhalb der Kapillaritätsgrenze, und der Wassergehalt des Sandes sinkt dabei auf 3,3 Vol.-Proz.

Sand von $1-0,5 \mathrm{~mm}$ (Kapillarität $131 \mathrm{~mm}$ ). Dieser Sand kann fast $10 \mathrm{~mm}$ Wasser oberhalb der Kapillaritätsgrenze $(131 \mathrm{~mm})$ festhalten. $20 \mathrm{~mm}$ Wasser sanken aber schon nach 2 Stunden unterhalb der Kapillaritätsgrenze, und der Wassergehalt des Sandes sank dabei bis auf 4,4 Volum.-Proz.

Sand von 0,5-0,2 $\mathrm{mm}$ (Kapillarität $246 \mathrm{~mm}$ ) kann sogar $30 \mathrm{~mm}$ Wasser oberhalb der Kapillaritätsgrenze $(246 \mathrm{~mm})$ bewahren. Wassergehalt dabei 18,4 Vol.-Proz. $40 \mathrm{~mm}$ sanken aber nach einem Tage unterhalb dieser Grenze, und der Wassergehalt ging zu 8 Vol.-Proz. nieder.

S a nd von $0,2-0,09 \mathrm{~mm}$ (Kapillarität $428 \mathrm{~mm}$ ).

$20 \mathrm{mmWass}$ sank. hier gleich $51 \mathrm{~mm}$ tief, nicht niedr., Wassergeh. dann $40 \mathrm{Vol}$.-Proz.

40 ,

60 ,

70 ,

80,

100

110

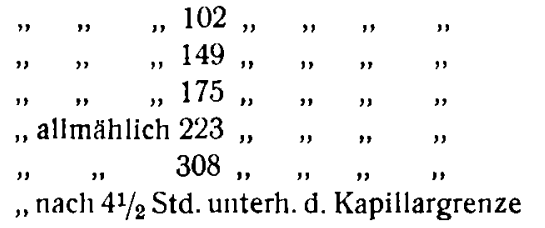

\begin{tabular}{|c|c|c|c|}
\hline ", & ", & 40 & ", \\
\hline " & $"$ & 40 & " \\
\hline " & ", & 40 & " \\
\hline$"$ & , & 30 & " \\
\hline " & dabei & $\begin{array}{l}33 \\
25\end{array}$ & " \\
\hline
\end{tabular}


Dieser Sand konnte darum, wenn er trocken war, $100 \mathrm{~mm}$ Wasser in der Oberfläche bewahren. Wenn das Wasser tiefer sank, war der Wassergehalt doch 25 Vol.-Proz.

Die Sandkörnungen von $0,09-0,04 \mathrm{~mm}$, von 0,04 bis $0,02 \mathrm{~mm}$ und von $0,02-0,009 \mathrm{~mm}$ verhielten sich sämtlich so, daß bei dem Begießen das Wasser anfangs die Sandporen vollständig oder fast vollständig füllte (Wassergehalt 40 Vol.-Proz.). Dann zog sich das Wasser allmählich tiefer, bis der Wassergehalt der Säule auf etwa 25 Vol.-Proz. gesunken war.

Die Sande feiner als $0,2 \mathrm{~mm}$ können daher sämtlich viel Wasser (25 Vol.-Proz.) in der Oberfläche halten. Die gröberen Sande können dagegen bei starkem Begießen nur 4-8 Vol.-Proz. Wasser aufbewahren. Die Grenze von 0,2 mm Körnerdurchmesser muß daher als die Grenze zwischen dem wasserlassenden und dem wasserhaltenden Sande aufgefaßt werden.

Um zu untersuchen, inwieweit dieses Resultat richtig sei, habe ich eine Reihe von Dünensanden und mit Kiefer und Heide bewachsenen Sanden auf ihren mittleren Körnerdurchmesser untersucht. Der mittlere Durchmesser wurde höchstens zu 0,6 mm gefunden und konnte auf $0,2 \mathrm{~mm}$ sinken. Dadurch wurde die oben festgestellte Lage der Grenze bestätigt.

\section{Das Verhalten der Sandkörner zu den Wurzelhaaren der Pflanzen.}

Man findet in botanischen Arbeiten die Angabe, daB die Wurzelhaare der Pflanzen einen Durchmesser von etwa $0,01 \mathrm{~mm}$ besitzen. Fs ist ganz klar, daß Wurzelhaare dieses Feinheitsgrades in die kleinen Zwischenräume zwischen Sandkörnchen von $0,01 \mathrm{~mm}$ Durchmesser gar nicht, und zwischen Körnern von 0,02 mm Durchmesser nur schwierig oder nicht eindringen können. Ein Boden, wo die Wurzelhaare zwischen den Bodenkörnern keinen Platz finden, muB in der Natur wie in der Kultur eine ganz andere Rolle für die Pflanzen spielen, wie die Böden, wo die Haare den Boden leicht durchdringen. Böden letzterer Art müssen sich wie leichte Böden verhalten, Böden erster Art müssen viel gründlicher bearbeitet, gelockert werden, um den Wurzelhaaren, welche die Nahrung aufsaugen, freie Ausbildung zu gestatten. Die Frage über das Verhältnis zwischen den Dimensionen der Wurzelhaare und Körnerdurchmessern der Sandarten ist daher einer näheren Untersuchung wert. 
Um unbeschädigte Wurzelhaare zu bekommen, wählte ich den Weg, Keimpflanzen mit neugebildeten Haaren zu untersuchen. Ich legte Samenproben einer Reihe von Kulturpflanzen zum Keimen aus. Wenn die Wurzelkeime Wurzelhaare gebildet hatten, bestimmte ich durch mikroskopische Messung den Durchmesser der Haare.

Es wurde gefunden, daß Weizen, Roggen und Gerste einen mittleren Durchmesser der Wurzelhaare von $0,008 \mathrm{~mm}$ zeigten. Die Futtergräser zeigten $0,008-0,010 \mathrm{~mm}$, der Hafer $0,010 \mathrm{~mm}$, die Kleearten 0,009-0,013, Erbse uud Wicke etwa 0,015, Rübensamen $0,007-0,010 \mathrm{~mm}$.

Ich suchte dann erst auf theoretischem Wege zu berechnen, in wie feine Sandkörnungen solche Wurzelhaare eindringen könnten. Das Resultat ${ }^{1}$ ) war, daß die Grenze für das Eindringen der Wurzelhaare für die Gräser bei Körnerdurchmesser von $0,02 \mathrm{~mm}$ und für Schmetterlingsblütler bei etwa $0,03 \mathrm{~mm}$ liegen sollte. Dann suchte ich die Frage durch das Experiment zu lösen.

Die Sandkörnungen von $0,2-0,002 \mathrm{~mm}$ wurden in sechs Gläser gefüllt, mit Wasser übergossen und der Wasserüberschuß weggenommen. Durch fleißiges Klopfen, bis sich kein Wasser mehr an der Oberfläche ansammelte, wurden die Sandarten zusammengeschüttelt.

Auf die wasserfreie Oberfläche der Sandkörnungen wurden Timothysamen gelegt. Die Gläser wurden mit Fließpapier bedeckt. Als die Samen gekeimt und die Keime Wurzelhaare gebildet hatten, zeigte sich beim Emporheben der Pflanzen, daß bei allen Sanden gröber als $0,02 \mathrm{~mm}$ Sand massenhaft den Wurzeln anhaftete. Bei den Sanden feiner als $0,02 \mathrm{~mm}$ haftete gar kein Sand den Wurzelkeimen an. Die Wurzelhaare hatten also nur in die Sandkörnungen, die gröber waren als $0,02 \mathrm{~mm}$, eindringen können.

Der Versuch wurde mit Kleesamen wiederholt. Es zeigte sich, daß in den Gläsern mit Sand von $0,2-0,09$ und $0,09-0,04 \mathrm{~mm}$ Sand in großer Menge den Wurzeln anhaftete. Bei dem Sande 0,04-0,02 $\mathrm{mm}$ haftete der Sand nur einzelnen Keimwurzeln an. Bei den feinen Sandkörnungen haftete kein Sand an den Keimwurzeln.

Die Berechnung und das Experiment hatten somit dasselbe Resultat geliefert. Die Grenze für das Eindringungsvermögen der Wurzelhaare in die Zwischenräume der Sandkörner liegt für die Gräser bei etwa $0,02 \mathrm{~mm}$ Durchmesser der Sandkörner, für die Schmetterlingsblütler ein wenig höher.

1) Landw. Versuchsstationen 69, 128 (1908). 
Die Koagulierbarkeit der feineren Sandkörnungen.

Durch H.Schulzel) ist es zuerst gezeigt worden, daß Kalkwasser Ton-Aufschlämmungen koaguliert, d. h., daß unter dem Einfluß des Kalkwassers die leichten Tonteilchen zu schweren Aggregaten zusammenhaften, welche $z u$ Boden sinken. Er fand die so gebildeten Tonabsätze bedeutend lockerer als die in reinem Wasser gebildeten. Daher der lockernde Einfluß des Kalkes auf die schweren Tone. Von Schlösing ${ }^{2}$ ) wurde weiter gefunden, daß auch Salzlösungen und Säuren den Ton koagulieren. Besonders stark koagulierend wirkten Kalksalzlösungen. Schon $0,2 \mathrm{~g}$ Kalk als lösliches Kalksalz war genügend, um ein Liter Tonwasser zu koagulieren. Verdünntere Alkalilösungen wirkten dagegen in entgegengesetzter Richtung. Konzentrierte Alkalilösungen koagulierten. Weitere Untersuchungen über das Koagulieren der Tone sind von Ad. Mayer, Hilgard ${ }^{3}$ ) und von Sachsze und Becker ${ }^{4}$ ) ausgeführt worden. Sachsze und Becker fanden in der Salzsäure das beste Koaguliermittel. Gipslösung, Salmiak und Ammonsulfat koagulieren ebenfalls rasch, Kochsalz, Salmiak und Chlorkalium wirkten ebenfalls gut koagulierend. Vitriole wirken schwächer. Monokaliumphosphat koaguliert nur langsam. Ton kann ebenfalls durch Kohlensäure koaguliert werden. Ferner zeigte G. B odlän d e ${ }^{5}$ ), daß die Elektrolyte Kaolinsuspensionen koagulieren, daß dagegen Nichtelektrolyte unwirksam sind. Die Reihenfolge der Klärfähigkeit ist annähernd die der Leitfähigkeit der Elektrolyte. Die Säuren, die Alaune und die Salze, die sich in Wasserlösung hydrolysieren (z. B. Kupfer- und Zinksalze) koagulieren am besten.

$\mathrm{Da}$ die Grenze zwischen Ton und Sand mir unklar schien, da die feinen mikroskopischen Sande von zahlreichen Verfassern den Tonen zugerechnet werden, und da die feinsten Sandkörnungen in mehrfacher Hinsicht die Eigenschaften der Tone anzunehmen scheinen, so stellte sich mir die Frage, ob die feineren Sande ebenfalls koagulierbar wären oder nicht. Zwar ist die Frage schon in einer Richtung beantwortet; denn amerikanische Forscher haben gezeigt, daß Kalkwasser mikroskopisch feine Sande koagulieren kann. ${ }^{6}$ ) Die Frage

1) H. Schulz e, Pogg. Ann. 129, 366 (1866).

2) Schlösing, Ann. d. Chim. et de Phys. [5] 2, 514 (1874).

3) Ad. Mayer u. Hilgard, Forschung. d. Agrik Phys. 2, 251 u. 441 (1879).

4) Sachsze und Becker, Landw. Versuchsstationen 43, 15 (1894).

$\left.{ }^{5}\right)$ Bodlä nder, Neues Jahrb. f. Mineralogie 2, 147 (1893).

$\left.{ }^{6}\right)$ U. S. Dep. of Agric., Division of soils, Bull. 5. Texture of some important soil formations 8, Plate 1 (1896). 
verdiente aber, ausführlicher behandelt zu werden, und ich habe darum eine Reihe von Versuchen darüber angestellt. Die Hauptresultate waren die folgenden.

Schon der Sand 0,04-0,02 $\mathrm{mm}$ zeigt eine Neigung, durch Einfluß von Kochsalzlösung und Salzsäure zu koagulieren. Deutlichere Koagulationserscheinungen zeigen sich erst bei den Sandkörnungen feiner als $0,02 \mathrm{~mm}$. Diese Sandkörnungen setzen sich in Salzlösungen viel schneller ab als in reinem Wasser. Eine deutlich sichtbare Flockenbildung wie bei dem Koagulieren der Tonaufschlämmungen zeigt sich jedoch nicht; auch nicht bei der feinsten von mir isolierten Sandkörnung von $0,002-0,001 \mathrm{~mm}$.

Aus reinem und aus schwach alkalischem Wasser setzen sich die feinen Sandkörnungen in dichtem, festen Zustande ab. Die Körnung $0,002-0,001 \mathrm{~mm}$ verhält sich ebenso. $15 \mathrm{~g}$ Sand nehmen dabei ein Volumen von nur $9,7-10 \mathrm{ccm}$ ein. Aus salzhaltigen Flüssigkeiten sind die Absätze weit voluminöser, können sogar einen 50 Proz. größeren Raum ausfüllen. Quellenwasser kann dieselben Resultate geben. Der in gesättigtem Kalkwasser gebildete Absatz kann sogar ein 24 Proz. größeres Volumen einnehmen.

Von diesen voluminösen, wasserreichen Absätzen sind die meisten mehr oder weniger flüssig. Nur der in gesättigtem Kalkwasser gebildete sehr voluminöse Absatz ist fest. Die flüssigen zeigen keine große Neigung, das überschüssige Wasser abzugeben und fest zu werden. Ein aus Gipslösung gebildeter Absatz hielt sich einen ganzen Monat völlig flüssig.

Diese Neigung der mikroskopischen Sande, sich flüssig zu erhalten, erklärt zum Teil die eigentümlichen Eigenschaften der nordschwedischen, hauptsächlich aus mikroskopischen Sanden bestehenden "Fließlehme" und der arktischen, ebenfalls gröbere Bestandteile enthaltenden und oft Steine und Blöcke tragenden „Fließböden". Die Fließlehme des Nordens bereiten den Eisenbahnbauten große Schwierigkeiten. Sie fließen aber nicht im Sommer, auch nicht bei starken Regenflüssen; nur beim Schneeschmelzen fließen sie. Die arktischen Fließböden fließen ebenfalls nur bei niedriger Temperatur. Nach Prof. J. G. Andersson ${ }^{1}$ ) fließen die meisten "stone-rivers" in den Falklands-Inseln nicht mehr, denn das Klima der Inseln ist jetzt wärmer, als es früher war.

I) J. G. Anders on, Solifluction, Journ. of Geology 14 (1906), und The Geology of the Falkland Islands, Schwed. Suidpolar-Expedition 1901-1903. 
Das Verhalten der Fließböden, nur bei niedrigen Temperaturen zu fließen, wird durch das allgemeine Verhalten der Böden in der Kälte erklärt. Simon Johansson ${ }^{1}$ ) hat darauf hingewiesen, dab, wenn ein nasser Boden friert, derselbe trocken wird, denn das Wasser ist jetzt in einen festen Körper übergegangen. Die gefrorene Bodenoberfläche muß dann durch die Kapillarkraft mehr Wasser von den unteren wasserreichen Bodenschichten anziehen. Dieses Wasser friert ebenfalls, und noch mehr Wasser wird angezogen. Die Bodenoberfläche muß dadurch große Mengen an Eis aufnehmen. Bei dem Auftauen des Eises muß dann die Bodenoberfläche leicht in halbflüssige Form übergehen. Wiederholtes Frieren und Auftauen machen die Böden noch flüssiger. Bei stark geneigter Bodenlage muß dann der aufgetaute, an Wasser überreiche Boden leicht in Flub kommen. Lockere Sandböden lassen jedoch das Wasser gleich in den Untergrund versinken. Die mikroskopischen Sandkörnungen haben aber, wie oben mitgeteilt ist, "wenig Neigung, das Wasser abzugeben. Dadurch erklärt es sich, daß gerade die an mikroskopischen Sanden reichen Böden bei niedrigen Temperaturen zu "Solifluktion" geneigt $\operatorname{sind}^{2}$ ).

Die Neigung der feinen Sande, sich aus reinem und salzhaltigem Wasser in flüssiger Form abzuscheiden, tritt nach meinen Versuchen schon bei der Sandkörnung 0,10-0,05 $\mathrm{mm}$ auf. Die aus reinem Wasser gebildeten flüssigen Absätze gehen schneller in feste Form über, als die aus salzhaltigen Wassern gebildeten.

Die Grenze, bei welcher die Salzlösungen einen stark koagulierenden Einfluß auf die feinen Sandkörnungen zu zeigen anfangen, liegt nach dem oben Angeführten bei der Korngröße von $0,02 \mathrm{~mm}$.

\section{Die Brown'sche "Molekularbewegung“.}

Der englische Botaniker R. Brown entdeckte im Jahre 1827, $\mathrm{da} B$ in Flüssigkeiten schwimmende sehr kleine Teile eine zitternde Bewegung besitzen. Exner zeigte, daß diese Bewegung durch Licht

1) Simon Johansson, Om fuktighetens fördelning och salpeterns vandring i lerjord (Upsala 1911).

$\left.{ }^{2}\right)$ Die Fließbodenerscheinungen sind von mir in meiner Abhandlung in den Int. Mitteil. f. Bodenkunde 1, 13, weiter behandelt. Eine ausführlichere Beschreibung der verschiedenartigen Fließbodenerscheinungen findet sich in der Abhandlung: Die geologische Bedeutung des Frostes von B. H ö g b o m, InauguralDissertation (Upsala 1914). 
und Wärme erhöht wird ${ }^{1}$ ), und daß Partikeln von der Größe von $0,004 \mathrm{~mm}$ die Bewegung nicht mehr zeigen. Schulze fand, daß, wenn die Teilchen durch Zusatz von Salzen und Säuren koaguliert werden, die Bewegung aufhört ${ }^{2}$ ). R. Zsigmondy zeigte 1905, daB diese Bewegung den kolloiden Körperchen eigentümlich ist ${ }^{3}$ ). In neuerer Zeit ist diese "B r o w n'sche Bewegung " von mehreren Forschern ausführlich studiert, so z. B. von The Svedberg $g^{4}$ ) und J. Perrin ${ }^{5}$ ).

Die feinste von mir isolierte Sandkörnung, 0,002-0.001 mm, zeigt unter dem Mikroskope diese Bewegung. Ich habe die obere Grenze der Bewegung festzustellen gesucht und gefunden, daß Sandkörner von $0,003 \mathrm{~mm}$ diese Bewegung nur schwach zeigen. Eine ausgeprägte Bewegung tritt erst bei Körnern von 0,002 mm Durchmesser ein. Später habe ich gefunden, daB die Grenze bei schuppenförmigen Mineralteilchen sich etwas höher stellt, bei 0,004 oder $0,005 \mathrm{~mm}$. Sie wechselt ebenfalls mit der Temperatur. Die Bewegung findet nur in reinem oder schwach alkalischem Wasser statt, Salze, Säuren und konzentrierte alkalische Lösungen hindern die Bewegung, koagulieren die Teilchen.

Wenn man die Brown'sche Bewegung als für die kolloiden Teilchen kennzeichnend auffabt, so muB man die obere Grenze der kolloiden Mineralteilchen bei der TeilchengröBe von $0,002(0,005) \mathrm{mm}$ ziehen. Sämtliche Mineralteilchen, die in der mechanischen Bodenanalyse nach der Absetzzeit von 8 Stunden (und bei $10 \mathrm{~cm}$ Wasserhöhe) abgeschlämmt werden, sind dann als kolloide Teilchen zu bezeichnen.

Die Hauptresultate dieser Untersuchung sind die folgenden.

Sandkörner gröber als $2,0 \mathrm{~mm}$ werden von dem Niederschlagswasser nur befeuchtet. Die Kapillaren werden hierbei nie gefüllt.

Sandkörnungen von $2,0--0,2 \mathrm{~mm}$ können, wenn anfangs trocken, etwas Wasser in den Kapillaren der Oberfläche festhalten. Bei stärkerer Wasserzufuhr zieht aber die Schwere der Wassersäulen das Wasser in den Untergrund herab, und die Körnungen behalten nur 3-8 Vol.-Proz.

1) Exner, Wiener Akad. Ber. [2] 56, 116; nach Jahresb. d. Chem. 1867.

$\left.{ }^{2}\right)$ Schulze, Pogg. Ann. 129, 368; nach Jahresb. d. Chem. 1867.

$\left.{ }^{3}\right)$ R. Zsigmondy. Zur Erkenntnis der Kolloide (Jena 1905), 108.

4) The Svedberg, Studien zur Lehre von den kolloiden Lösungen (Upsala 1907).

s) J. Perrin, Kolloidchem. Beih. 1, 221 (1910); siehe auch „Die Atome“ (Dresden 1914). 
Wasser. Diese Sandkörnungen bilden daher trockene Böden. Sie eignen sich nur als Waldböden.

Sandkörnungen feiner als $0,2 \mathrm{~mm}$ liefern bessere Böden. Schon die Körnung 0,2 $0,09 \mathrm{~mm}$ kann, wenn anfangs trocken, $100 \mathrm{~mm}$ Wasser in die Oberfläche aufnehmen. Wenn das Wasser durch die Kraft der Kapillaren tiefer sinkt, bleibt doch meistens 25 Vol.-Proz. Wasser in den oberen Schichten zurück.

Sand gröber als $0,02 \mathrm{~mm}$ zeigt in Zimmerluft gar keine Hygroskopizität. Feinere Sande sind hygroskopisch. Sand gröber als $0,02 \mathrm{~mm}$ trocknet zu losem Pulver ein. Sand feiner als $0,02 \mathrm{~mm}$ bildet bei dem Austrocknen lose Kuchen, die um so fester werden, je feiner der. Sand ist. Kompakter nasser Sand feiner als 0,02 $\mathrm{mm}$ läßt die Wurzelhaare der Pflanzen zwischen die Sandkörner nicht eindringen, denn die Sandporen sind für die Wurzelhaare zu klein. Sand feiner als $0,02 \mathrm{~mm}$, in Wasser aufgeschlämmt, fängt an, bei Zusatz von Salzlösungen oder Mineralsäurelösungen koaguliert zu werden.

Bei etwa $0,002 \mathrm{~mm}$ (oder etwas höher) liegt die Grenze der Brown'schen Bewegung für Teilchen der gewöhnlicheren Mineralien. Ehrenberg hat dazu darauf aufmerksam gemacht, daß die Bakterien, deren mittlere Größe $0,001 \mathrm{~mm}$ beträgt, zwischen dicht gelagerten Teilchen fciner als $0,002 \mathrm{~mm}$ schwerlich Platz finden können'). Nach meinen Untersuchungen liegt hier ebenfalls die obere Grenze der Plastizität bei den tonbildenden Mineralien.

Aus diesen Ergebnissen muB manden SchluBziehen, $d a B$, wenn maneine rationelle Klassifikationder Bodenkörner aufstellen will, die Grenzen der Körnerklassen bei den Körnergrößen von $2,0 \mathrm{~mm}, \cdots 0,2 \mathrm{~mm}, \ldots 0,02 \mathrm{~mm}$ und bei $0,002 \mathrm{~mm} z u$ ziehen sind.

Vorläufiger Beschluß der Agrogeologen.

Die zweite Agrogeologen-Konferenz in Stockholm 1910 hatte eine Kommission ernannt mit dem Verfasser als Vorsitzenden, welche Vorschiäge zu internationalen Methoden der mechanischen Bodenanalyse ausarbeiten und der nächsten Konferenz vorlegen sollte. Nachdem die brieflichen Verhandlungen der Kommission in fünf gedruckten Mitteilungen von dem Vorsitzenden den Kommissions-Mitgliedern vorgelegt waren, hatte die Kommission am 31. Oktober 1913 eine Sitzung in Berlin mit Geheimrat Wanschaffe als Vorsitzenden und

1) Ehrenberg, Koll.-Zeitschr. 2, 206 (1908). 
Dr. Schucht als Schriftführer. Es wurde dabei beschlossen, die obengenannte Einteilung der Bodenkörner zum internationalen Gebrauch zu befürworten. Für die deutsche Sprache wäre vorläufig die folgende Nomenklatur anzuwenden.

$\begin{array}{cll}\text { Körner größer als } 20 \mathrm{~mm} & \text { Stein und Geröll } \\ " & \text { von } 20-2 \mathrm{~mm} & \text { Kies } \\ " & n 2-0,2 \mathrm{~mm} & \text { Grobsand } \\ " \quad & \Rightarrow 0,2-0,02 \mathrm{~mm} & \text { Feinsand } \\ " \quad \text { " } 0,02-0,002 \mathrm{~mm} & \text { Schluff } \\ " \quad \text { feiner als } 0,002 \mathrm{~mm} & \text { Kolloide Teilchen oder Rohton. }\end{array}$

\section{Die Methoden der mechanischen Bodenanalyse.}

Die mechanische Bodenanalyse sucht die Bodenbestandteile nach der verschiedenen Größe der Bodenkörner zu trennen. Die gröberen Bodenbestandteile werden durch Siebe getrennt. Die feineren werden durch Wasser von den gröberen abgeschlämmt und dann durch systematisches Schlämmen weiter getrennt.

Die Schlämmethoden sind vielerlei $\left.\operatorname{Art}^{1}\right)$. Sie können in drei Gruppen eingeteilt werden.

Erst die Spülapparatmethoden, wo die Bodenteile durch strömendes Wasser getrennt werden. Je schneller der Wasserstrom, desto gröbere Teile werden abgeschlämmt. Methoden dieser Art sind die von Nöbel (1864), E. Dietrich (1866), E. Schöne (1867), E. W. Hilgard (1873) und J. Kopecky (1901).

Dann die Sedimentiermethoden, bei welchen der Boden mit Wasser aufgerührt wird, das Wasser eine Zeit in Ruhe gelassen wird und dann die nicht zum Boden gesunkenen Teilchen durch Absiphonieren von dem Bodensatze getrennt werden. Durch mehrmalige derartige Behandlung wird das Trennen quantitativ. Durch Benutzen von verschiedenen Ruhezeiten und verschiedenen Wasserhöhen lassen sich Schlämmprodukte verschiedener Feinheitsgrade darstellen. Solche Schlämmethoden sind die von W.Knop (1873), J. Kühn, Bennigsen und Clausen, die von Th.Schlösing, W. Williams-Fadejeff, G. Appiani und A. Atterberg.

Weiter die Zentrifugmethoden. So die von dem amerikanischen Bureau of Soils benutzte Zentrifugalmaschine, P. A. Yoder's Centrifugal Elutriator und B. Sjollema's Methode.

1) Siehe J. Kö n ig, Untersuchung landw. und gewerbl. wichtiger Stoffe, u. H. W. Wile y, Principles and practice of agricult. analysis, vol. 1, soils 200-273 (1906). 
Durch die Spülapparate kann man nur Bodenkörner gröber als $0,05 \mathrm{~mm}$ trennen. Durch die Sedimentierapparate kann man Teilchen bis zu dem Feinheitsgrade von $0,01 \mathrm{~mm}$ abscheiden. Es scheint mir, daß man durch die Zentrifugmethoden noch feinere Teilchen quantitativ trennen könne. Versuche in dieser Richtung sind aber bisher nicht gemacht.

Der von mir konstruierte Schlämmapparat ist ein Sedimentierapparat. Er hat die nachstehende form.

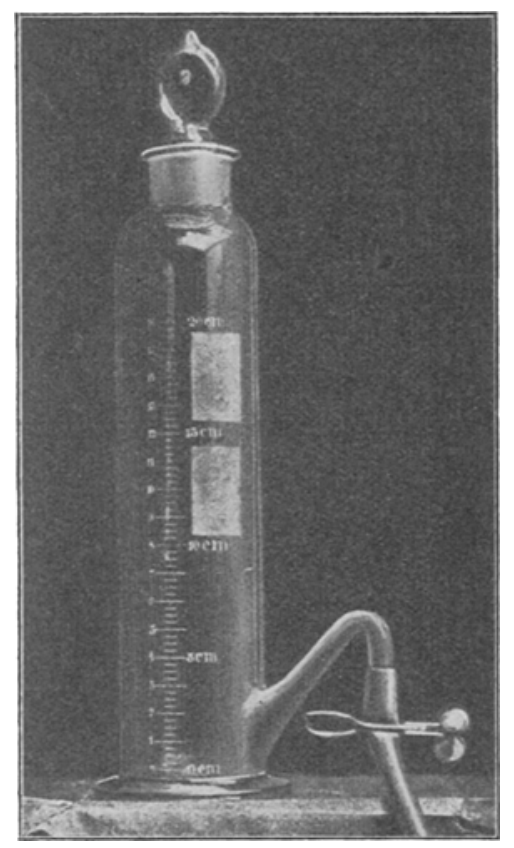

Fig. 1

Bei der obengenannten Sitzung der internationalen Kommission für die mechanische Bodenanalyse wurde beschlossen, meinen Schlämmapparat als Normalapparat zu empfehlen. Andere Apparate wären so einzustellen, daß sic dieselben Resultate geben als mein Apparat bei Befolgung der von mir gegebenen Vorschriften.

Man kann auch ohne alle Apparate systematisch schlämmen. Auf Seite 60 habe ich die für das Gewinnen von bestimmten Teilchengrößen nötigen Vorschriften gegeben. Die Vorschriften gelten sowohl für sämtliche Sedimentierapparate, wie für das Schlämmen ohne Apparate.

Vor dem Schlämmen muß man die in den Böden vorkommenden Körneraggregate erst genau zerteilen. Das geschieht am besten durch 
langes Kochen des Bodens mit Wasser und durch längeres Schütteln mit Wasser in besonderen Schüttelmaschinen. Der Engländer W. B e a m (in Khartoum) hat ferner nachgewiesen, daß ein anhaltendes Bearbeiten der mit wenig Wasser gemischten Bodenproben vermittels eines steifen Malerpinsels für das Zerteilen der Aggregate besonders guten Dienst liefert. Ich habe dieses Verfahren aufgenommen. Bei schwereren Böden muß während der Schlämmanalyse das Durchbürsten der Böden mit ein wenig Wasser zwei- bis dreimal wiederholt werden.

Ueber die übrigen für den Gebrauch meines Apparates gegebenen Vorschriften muB ich zu meiner Abhandlung Internat. Mitt. f. Bodenkunde 2, 314-324 (1912) verweisen.

Das Trennen der Bodenteilchen durch Schlämmen mit Wasser setzt voraus, daß die verschiedenen Bodenmaterialien fast dasselbe spezifische Gewicht besitzen. Die wichtigeren Bodenmaterialien zeigen auch ziemlich übereinstimmende spezifische Gewichte, z. B. Quarz 2,5-2,8, Feldspäte 2,53-2,75, Kaliglimmer 2,76-3,1, Kaolinit 2,2-2,6, Biotit 2,8-3,2, Hornblende 2,9-3,3. Sehr eisenreiche Mineralien zeigen jedoch viel höhere spezifische Gewichte, z. B. Limonit etwa 3,6, Magnetit 4,9-5,2 und Hämatit 5,19-5,28. Limonit und Hämatit finden sich aber nur in den feinsten Schlämmprodukten, und Magnetitkörner spielen in den Böden keine grote Rolle. Bei den Verschiedenheiten der spezifischen Gewichte der Bodenmineralien ist es doch klar, daß die mechanische Bodenanalyse die Bodenkörner nach der Körnergröße nur annähernd trennen kann. Es hat darum keinen Zweck, eine große Reihe von Körnergruppen aufzustellen. Ich unterscheide daher meistens nur die auf Seite 74 genannten größeren Körnergruppen und finde es nur bei Feinsandböden bisweilen vorteilhaft, den Feinsand weiter einzuteilen.

Die Humusteilchen der Böden zeigen ein sehr niedriges spezifisches Gewicht und erschweren sehr das Reindarstellen der mineralen Körnergruppen Bei humusreichen Böden muß man daher die Humusteile vor dem Schlämmen zerstören. Ich zerstöre bei solchen Böden die Humusstoffe durch zwölfstündige Behandlung der Bodenproben mit kalter Bromlauge.

Die Verwendbarkeit der mechanischen Bodenanalyse für die Klassifikation der Bodenarten.

Die von mir ausgeführten zahlreichen mechanischen Bodenanalysen haben in dieser Hinsicht folgendes ergeben. 
Sämtliche Böden, die bei der mechanischen Analyse höheren Gehalt an "Kolloiden Teilchen" als 50 Proz. gezeigt haben, zeigten sich als hochplastische, sehr schwere Tonböden.

Sämtliche Böden, die höheren Gehalt an Grobsand als 50 Proz. zeigten, waren trockene, nur für Waldkultur passende Sandböden.

Die Böden aber, die mehr als 50 Proz. an Feinsand oder an Schluff zeigten, haben sich bald als "lehmige Tone“, bald als "normale Lehme", bald als "lehmige Sandböden" erwiesen.

Wie schon oben gesagt ist, genügt daher die mechanische Bodenanalyse nicht, um die Böden korrekt zu klassifizieren. Die physikalischen Eigenschaften der Böden müssen bei der Klassifikation notwendig in Betracht gezogen werden.

\section{Die Plastizităt der Tone.}

Bei der oben beschriebenen Untersuchung hatte ich nur die Eigenschaften der sandartigen Bodenbestandteile behandelt. Die tonartigen Bestandteile waren aus dem Untersuchungsmateriale durch das Behandeln mit Salzsäure und Natronlauge wie durch das Schlämmen entfernt. Die Eigenschaften der tonartigen Bodenbestandteile waren aber jetzt $z u$ bestimmen. Ich hatte mir das Ziel gestellt, eine auf die Bodeneigenschaften gegründete Klassifikation der Böden aufzustellen. Und es mußten daher nicht nur die Eigenschaften der sandigen, sondern ebenfalls die Eigenschaften der tonigen Bodenbestandteile ausführlich stúdiert werden.

Ueber die chemische Natur der tonartigen Bodenbestandteile weiß man nicht viel ganz Sicheres. Man hat früher den Begriff Ton mit dem Begriffe Kaolin oder Kaolinit gleichgestellt. Später hat man die salzsäurelöslichen Tonbestandteile als Zeolithe erklärt. Kaolinit und Zeolithe können zwar in den Tonen vorkommen; sie sind aber nicht die einzigen Tonbestandteile. Da die Bestandteile der Tone nicht genügend bekannt sind, die Eigenschaften der Tone aber zu ihrer Art wohl gekannt sind, so geht daraus hervor, daß der Begriff Ton kein chemischer, sondern ein physikalischer Begriff , sein muß.

Die Eigenschaften der Tone sind vielerlei Art. Recht viele dieser Eigenschaften haben sich bei der Entwickelung der Lehre von den Kolloiden als Kolloideigenschaften erwiesen. So das Koagulieren der Tonaufschwemmungen durch Salze und Säuren. So die von den Agrikulturchemikern in den Jahren $1850-1880$ so eifrig studierten Adsorptions- 
verhältnisse der tonartigen Bodenbestandteile; ferner die Brown'sche Bewegung der Tomeilchen.

Wenn man die obere Grenze der kolloiden Dimensionen bei der Grenze der Brown'schen Bewegung zieht, so gehören sämtliche tonartigen Bodenbestandteile $\mathrm{zu}$ den Kolloiden und die Tonbodenkunde muß dann völlig innerhalb des Gebietes der Kolloidlehre fallen.

Die Haupteigenschaften der Tone sind ihre Plastizität oder Formbarkeit im nassen Zustande und ihre feste bis harte Konsistenz in trockener Form. Plastizität und hohe Konsistenzgrade sind deshalb Eigenschaften, die einigen Gruppen von wasserarmen Kolloidsystemen zukommen. lch will hier nur die Plastizitätsverhältnisse der Tone näher studieren.

\section{Was ist Plastizität?}

Plastizität bedeutet Formbarkeit.' H. Seger hat folgende Definition des Plastizitätsbegriffes gegeben ${ }^{1}$ ):

Unter Plastizität versteht man die Eigentümlichkeit fester Körper, in ihre Poren eine Flüssigkeit aufzunehmen und damit eine Masse zu bilden, der durch Kneten und Drücken jede beliebige Form gegeben werden kann und schlieblich nach dem Aufhören des Druckes und nach der Entfernung der Flüssigkeit dieselbe auch als fester Körper unverändert zu bewahren.

Nach dieser Definition müssen plastische Stoffe sich nicht nur im feuchten Zustande gut formen lassen, sie müssen ebenfalls die ihnen gegebene Form sowohl im feuchten wie im trockenen Zustande völlig bewahren. Feuchter, feiner Sand und feuchter Fließlehm (mikroskopischer Sand) lassen sich wohl formen, sind aber gleichwohl nicht plastisch. Der geformte Sand zerfällt beim Trocknen. Der Fließlehm ist, wenn formbar, halbflüssig, und kann die Form nicht bewahren.

Man bezeichnet gern ebenfalls solche Stoffe als plastisch, die schon in unvermischter Form plastisch sind. Als Beispiele derartiger Stoffe sind zu nennen zunächst das Metall Natrium, welches sich bei gewöhnlicher Temperatur zu Drähten auspressen läßt, dann das Eisen und mehrere andere Metalle, welche sich bei höherer Temperatur zu Drähten ziehen und zu Platten auswalzen lassen, schließlich viele Gesteinsarten, die, weil sie bei hohem Seitendrucke gefaltet werden, von den Geologen als plastisch erklärt werden. Diese Arten von Plastizität können als Plastizität im weiteren Sinne bezeichnet werden. Unter Plastizität im beschränkteren Sinne verstehe ich die Eigen-

1) C. Bisch off, Die feuerfesten Tone. 3. Aufl. (Leipzig 1904), 22. 
schaft der Körper, sich unter den Fingernzu Drähten ausrollen zu lassen. Die Tone besitzen in feuchtem Zustande Plastizität im beschränkteren Sinne.

Die Methoden zur Bestimmung der Plastizität.

In meiner oben zitierten Abhandlung: „Die Plastizität der Tone“ habe ich 20 teils qualitative, teils quantitative Plastizitätsbestimmungsmethoden beschrieben. Keine derselben hat sich jedoch als völlig befriedigend bewährt. Bei den Methoden der Tontechniker knetet man den Ton mit so viel Wasser durch, daß die Masse gerade nicht mehr an den Händen klebt. Die Masse ist jetzt "handgerecht", hat jetzt diejenige Konsistenz, bei welcher der Ton im Tonschneider verarbeitet werden soll. Mit einem solchen "Normalteige" werden die Untersuchungen gemacht. Bald wird bestimmt, wie weit ein Tonballen sich ausrollen läßt, ohne zu zerreißen. Bald wird aus einer Handpresse ein freihängender Tonfaden herausgetrieben, bis er durch sein eigenes Gewicht abreibt. Bald wird ein zylindrischer Probekörper von oben gepreBt, bis Rißbildung erfolgt, usw. Es wird jedoch von keiner der vielen Methoden gesagt, daß sie die wirkliche Plastizität richtig angibt.

Daher hat man die Plastizität auch auf vielerlei anderen Wegen bestimmen wollen. P. Jochum und W. Olschewsky wollen die Plastizität aus der Festigkeit des getrockneten Tones berechnen. C. Bischoff hat die Plastizität aus der Hygroskopizität des Tones zu berechnen versucht. J. Aron, F. Tschaplowitz, E. Van der Bellen und M.Simonis wollen die Plastizität aus der Wasseraufnahmefähigkeit des Tones bestimmen. A. Sokoloff bestimmt die Geschwindigkeit des Zerfallens der Tone im Wasser. H. E. A shley bestimmt, wie viel Farbstoff das Tonpulver aus Lösungen von Malachitgrün oder Brillantgrün aufnehmen kann. C. Bischoff bestimmt, wieviel Sand die Tone aufnehmen können (das Bindevermögen), ohne beim Trocknen die Festigkeit zu verlieren. Sämtliche Methoden wollen die Plastizität bei einem Zustande des Tones bestimmen, in dem der Ton nicht plastisch ist, und sie können daher keine zuverlässigen Resultate liefern. Die Plastizitätserscheinungen müssen daher viel ausführlicher als bisher studiert werden, damit es gelingen könne, eine fehlerfreie Methode der Plastizitätsbestimmung aufzustellen. Ich habe daher ein näheres Studium der Plastizitätserscheinungen aufgenommen. 
Die erst angenommenen Plastizitätsgrenzen.

Wenn man einen schweren Ton im gepulverten Zustande mit vicl Wasser ausrührt, bekommt man einen flüssigen Tonbrei. Bei weniger Wasser wird der Brei dickflüssig. Durch Verdunsten des Wassers geht dann der Brei in eine zähe, klebrige Masse über, die an den Fingern, an Holz und Metallen klebt. Wegen des schmierigen, klebrigen Zustandes wird der Ton noch nicht als plastisch anerkannt. Bei weiterem Trocknen verschwindet dann die Klebrigkeit. Der Ton läßt sich jetzt gut formen, hat jetzt die für die Tonarbeiten vorgeschriebene "Normalkonsistenz" und wird plastisch und "handgerecht" genannt. Bei noch fernerem Austrocknen hört schließlich die Formbarkeit auf. Der Ton ist zwar noch feucht, die Tonstücke können jetzt aber nur durch Druck zu zusammenhängenden Massen vereint werden. SchlieBlich vergeht auch diese Formbarkeit: der Ton wird fest und bei völlig trockenem Zustande hart.

Die verschiedenen Tone zeigen indessen nicht ganz dieselben Eigenschaften. Der eine Ton hält sich länger klebend, der andere klebt nur wenig und läbt sich leichter formen. Um diese Verschiedenheiten in Ziffern ausdrücken zu können, muB man die Grenzen aufsuchen, wo die Eigenschaften sich ändern. Wenn der eine Ton zwischen den Wassergehaltsgrenzen von 60 und 40 Teilen Wasser, auf 100 Teile Ton gerechnet, gut formbar ist, der andere aber nur zwischen 40 und 35 Teilen Wasser formbar ist, so zeigen doch die beiden Tone sehr verschiedene Eigenschaften. Es hat somit für das Studium der Tone große Bedeutung, die Grenzen aufzusuchen, wo die Eigenschaften sich ändern. Ich habe gefunden, daß folgende wichtigere Grenzen sich aufstellen lassen.

1. Die obere Grenze der Dickflüssigkeit, das ist die Grenze, wo ein dickflüssiger Tonbrei durch Wasseraufnahme so wasserreich wird, dab er fast wie Wasser fliebt.

2. Die FlieBgrenze; die Wassergehaltsgrenze, bei welcher zwei Stückchen Tonbrei, in eine Schale gelegt, beim heftigen Stoben der Schale nicht mehr ineinanderfließen wollen.

3. Die Klebegrenze; die Grenze, bei welcher der Ton nicht mehr klebt.

4. Die Ausrollgrenze; die Grenze, wo der Tonteig sich nicht mehr zu Drähten ausrollen läßit.

5. Die Schwindungsgrenze (deren Bedeutung erst später gefunden wurde). 
Die Lagen der Grenzen werden durch Wassergehaltsbestimmungen festgestellt.

Da die Klebegrenze die Grenze der obengenannten "Normalkonsistenz" ist, und die Tone bei dieser Grenze in der Industrie als "handgerecht" bezeichnet werden, wurde diese Grenze als die obere (wasserreichere) Plastizitätsgrenze angesehen. Die Ausrollgrenze wurde als die untere Plastizitätsgrenze aufgefaßt. Diese beiden Grenzen würden das Plastizitätsgebiet begrenzen.

Nachdem ich die genannten fünf Grenzen aufgestellt hatte, untersuchte ich die Lage der vier ersten Grenzen bei einer Reihe von 75 Ton- und Lehmproben. Die Grenzen wechselten zwischen den folgenden Wassergehaltsziffern:

Die Grenze der Schwerflüssigkeit zwischen 168 und 39

Die Lage der Fliehgrenze zwischen . . . 75 und 25

Die Lage der Klebegrenze zwischen . . 72 und 24

Die Lage der Ausrollgrenze zwischen . . 49 und 24

Die Fliebgrenze und die Klebegrenze wechselten somit zwischen fast denselben Ziffern. Ich wurde dadurch veranlaßt zu untersuchen, ob diese Grenzen so geändert werden könnten, daß sie identische Ziffern lieferten. Ein solches Justieren der Grenzbestimmungsmethoden wurde aber als unmöglich befunden. Bald lag die Klebegrenze niedriger, bald lag sie höher als die Fließgrenze. Daß die Klebegrenze, welche die obere Plastizitätsgrenze darstellen sollte, eine höhere Lage (einen höheren Wassergehalt des Bodens) als die Fließgrenze besitzen konntc, war mir ganz unerwartet. Bei höheren Wassergehalten als die der Fließgrenze muß ja der Boden halbflüssig sein, und er könnte dann wohl nicht als plastisch aufgefaßt werden.

Noch ein Resultat der obengcnannten Ziffern war mir sehr schwer verständlich. Obere und untere Plastizitätsgrenzen müssen ja nur bei plastischen Böden unterschieden werden können. Bei nicht plastischen Böden - Lehmböden - müssen dagegen die beiden Grenzen zusammenfallen. Die Klebegrenze und die Ausrollgrenze, welche die beiden Plastizitätsgrenzen darstellen sollten, wollten aber bei meinen Lehmproben gar nicht zusammenfallen.

Bei der Behandlung der Frage waren darum notwendigerweise ein oder mehrere Fehler begangen. Wo waren aber diese Fehler zu finden? 
Die Plastizitätsklassen.

Um dieser schwierigen Frage näher zu kommen, beschlob ich, die Methode C. Bischoff's für die Bestimmung des Bindevermögens zu versuchen, denn diese Methode war die einzige der älteren Plastizitätsbestimmungsmethoden, zu der ich Zutrauen hatte. Die Methode bestimmt, wieviel Sand die Tone aufnehmen können, ohne beim Austrocknen die Festigkeit zu verlieren. Ich beschlob, dieselbe so zu verändern, daß ich untersuchte, wieviel Sand die Tone aufnehmen können, ohne die Plastizität zu verlieren. Je mehr Sand der Ton aufnehmen konnte, als um so höher muB die Plastizität des Tones angesehen werden. Zur Vereinfachung der Frage wurden nur zwei Sandmischungen benutzt. Die Tone, welche zwei Gewichtsteile Sand aufnehmen konnten, ohne dabei die Plastizität zu verlieren, wurden als der ersten Plastizitätsklasse zugehörend aufgefaBt. Die Tone, welche nur eine Zumischung von der gleichen Menge Sand erlaubten, wurden in die zweite Plastizitätsklasse gestellt. Die, welche bei der Zumischung von der gleichen Menge Sand die Plastizität verloren, wurden als der dritten Plastizitätsklasse zugehörend angesehen ${ }^{1}$ ). (Die Plastizitätsklassen würden richtiger Bindefähigkeitsklassen genannt werden.)

Bei diesen Untersuchungen wurde das folgende Verfahren benutzt. Der feingepulverte Ton, welcher ein Sieb von $0,2 \mathrm{~mm}$ passiert hatte, wurde teils mit der gleichen Menge und teils mit der doppelten Menge Sand vermischt. Diese Mischungen wurden dann auf Plastizität in der folgenden Weise untersucht. Erst wurde so viel Wasser zugeführt, daB die Fliekgrenze erreicht wurde, dann wurde der so gewonnene Tonteig auf Ausrollbarkeit geprüft. War der Teig nicht ausrollbar, so wurde die Mischung als nicht plastisch angesehen. Es wurde gefunden, daß die Korngröße des angewandten Sandes nicht ohne EinfluB auf die Resultate war. Daher wurde stets Glazialsand von 0,3 bis $0,1 \mathrm{~mm}$ Körnerdurchmesser benutzt.

\section{Die wirklichen Plastizitätsgrenzen.}

Nach der so veränderten Bis ch off'schen Methode wurden jetzt 19 Tone untersucht. Die Resultate, die dabei gewonnen wurden, führten endlich Klarheit in der Frage herbei. Es wurde gefunden,

1) Es that sich spăter gezeigt, daß gewisse Tone aus siidlicheren Ländern sogar drei Gewichtsteile Sand binden können. Ich habe dann für solche Tone eine Extra-Plastizitatsklasse aufgestellt. 
daB bei den verschiedenen Plastizitätsklassen der Tone die drei Grenzen, die Fließgrenze, die Klebegrenze und die Ausrollgrenze, die gegenseitige Lage wie in dem folgenden Diagramme zeigten:

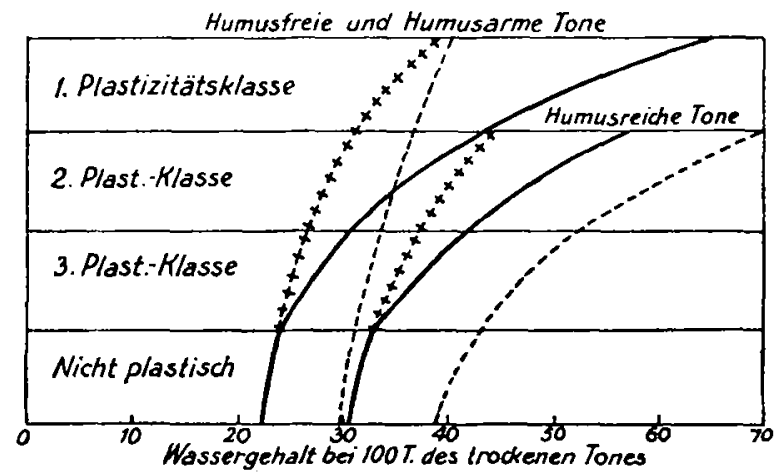

Fig. 2. In dem Diagramme bedeuten

$$
\begin{aligned}
& - \text { die Fließgrenze, } \\
& ------- \text { die Klebegrenze, } \\
& ++++++++ \text { die Ausrollgrenze. }
\end{aligned}
$$

Das Diagramm zeigt, daß die Fließgrenze und die Ausrollgrenze bei den nichtplastischen Böden zusammenfallen, bei den Tonen aber sich um so mehr voneinander entfernen, in je höhere Plastizitätsklassen die Tone sich einordnen. Diese beiden Grenzen verhalten sich demnach, wie wirkliche Plastizitätsgrenzen sich verhalten müssen. Die Klebegrenze hat einen ganz anderen Verlauf. Bei den humusfreien und humusarmen Tonen der höchsten Plastizitätsklasse nähert sich diese Grenze immer mehr der Ausrollgrenze, um schließlich mit derselben fast zusammenzufallen. Bei den Tonen niedriger Plastizität und bei den nichtplastischen Böden wollen die beiden Grenzen gar nicht zusammenfallen. So können sich aber zwei Plastizitätsgrenzen, die zwischen sich das Plastizitätsgebiet einschließen sollen, nicht verhalten. Die Klebegrenze kann daher unmöglich die obere Plastizitätsgrenze sein, sondern es muß die Fließgrenze die obere Plastizitätsgrenze darstellen. Die Fliebgrenze und die Ausrollgrenze müssen somit als die beiden Plastizitätsgrenzen angesehen werden.

Da aber die Klebegrenze bei den Tonen der ersten Plastizitätsklasse zwischen den beiden wirklichen Plastizitätsgrenzen ihre Lage hat, so muB man bei diesen Tonen zwei Arten von Plastizität unterscheiden: die klebende Plastizität und die nichtklebende Plastizität. 
Man hat die Tone in dem Zustande, wo sie kleben, nicht als plastisch ansehen wollen. Daß sie aber auch im klebenden Zustande als plastisch angesehen werden müssen, belehrt uns der plastische Mehlteig der Bäcker. Die Finger und der Tisch müssen mit Mehl überstreut werden, damit man den stets klebenden Teig behandeln kann. Jedoch muh der klebende Teig als plastisch angesehen werden.

Gerade die Auffassung der Klebegrenze als eine obere Plastizitätsgrenze ist die Hauptursache gewesen, weswegen die Plastizitätserscheinungen so schwierig $\mathrm{zu}$ verstehen waren.

Meine Bestimmung des Plastizitätsbegriffes.

Die Ausrollbarkeit ist für mich das beste qualitative Kennzeichen der Plastizität. Die Ausrollbarkeit muß aber unterhalb (bei niedrigerem Wassergehalt als die) der Fließgrenze stattfinden. Die feinkörnigeren Lehme können eine Ausrollbarkeit oberhalb der Fließgrenze zeigen; sie sind aber dabei halbflüssig oder schwerflüssig. Da aber als Hauptunterschied zwischen den Tonen und Lehmen allgemein angegeben wird, daß die Tone plastisch und die Lehme nicht plastisch sind, so scheint es nicht angemessen, von einer bei den Lehmen vorkommenden und oberhalb der Fließgrenze liegenden Plastizitätsform zu sprechen. Ich will daher den Plastizitätsbegriff für die Tone in folgender Weise definieren :

Plastisch sind die Böden, die bei Wassergehalten, niedriger als die Fliebgrenze, sich zu Drähten ausrollen lassen.

Diese Definition liefert eine qualitative Methode, um die Böden auf Plastizität $z$ u untersuchen. Man setze dem Bodenpulver so viel Wasser zu, daß die Fliebgrenze erreicht wird, und untersuche dann, ob der Bodenteig ausrollbar ist.

Die Plastizitätszahlen.

Ich habe oben die Tone in drei (oder vier) Plastizitätsklassen (Bindigkeitsklassen) eingeteilt. Die Tone lassen sich aber ebenfalls nach den Plastizitätszahlen einteilen. Unter den Plastizitätszahlen verstehe ich den Abstand zwischen den Plastizitätsgrenzen. Wenn bei einem Tone die obere Plastizitätsgrenze (die Fliebgrenze) bei einem Gehalte von 44 Teilen Wasser (auf 100 Teile des trockenen Tones gerechnet) liegt, und die andere Plastizitätsgrenze (die Ausrollgrenze) bei 23 Teilen Wasser liegt, so gibt die Differenz $44-23$ die Plastizitätszahl $=21$. 
Bei einem anderen Tone können die Plastizitätsgrenzen bci den Ziffern 31 und 21 liegen. Die Plastizitälszahl ist dann $=10$.

Die Plastizitätszahlen zeigen die Begrenzung des Plastizitätsgebietes bei jedem Tone. Sie liefern daher eine bequeme Methode, die Tone näher zu klassifizieren. Die Plastizitätszahlen können von 1 bis zu 40 wechseln. Bei der dritten oben aufgestellten Plastizitätsklasse kommen Plastizitätszahlen von 1 bis 7 vor; bei der zweiten Plastizitätsklasse habe ich Zahlen von 7 bis 15 gefunden; bei der ersten wechseln die Zahlen von 17 bis 27 . Die höchsten Plastizitätszahlen gehören in die "Extraklasse".

\section{Die verschiedene Zähigkeit der Tone.}

Die Zähigkeit der Tone innerhalb des Plastizitätsgebietes ist sehr wechselnd. Sie ist niedrig bei der Fließgrenze, kann aber bei der Ausrollgrenze sehr hoch sein. Die Zähigkeit ist daher nur bei bestimmten Wassergehalten festzustellen. Die Zähigkeit in der Nähe der Ausrollgrenze ist bei den Tonen von niedrigen Plastizitätszahlen niedrig, kann aber bei den Tonen hoher Plastizitätszahlen so hoch steigen, daß der Tonteig bei dem Versuche, denselben auszurollen, viel Widerstand leistet. Die Zähigkeitsziffern der Tone können sehr wechselnd sein, und man hat daher die Zähigkeitsbestimmung als eine Methode zur Bestimmung des "Plastizitätsgrades" anwenden wollen. Die Zähigkeitsziffern sind aber Konsistenzzahlen und sollen als solche später behandelt werden ${ }^{\prime}$.

\section{Der Plastizitätsgrad.}

Wir kommen jetzt zu der schwierigen Frage, wie der "Plastizitätsgrad " zu bestimmen ist. Was ist aber unter dem Begriffe Plastizitätsgrad zu verstehen? Plastizität ist Formbarkeit oder Ausrollbarkeit. Plastizitätsgrad wäre somit Formbarkeitsgrad oder Ausrollbarkeitsgrad. Alle plastischen Böden sind aber innerhalb des Plastizitätsgebietes gut formbar. Schlechte Formbarkeit findet man nur bei den niedrigsten Plastizitätszahlen, da die Tone schon durch schwaches Austrocknen die Plastizität verlieren. Schlechte Ausrollbarkeit findet sich ebenfalls nur bei niedrigen Plastizitätszahlen, denn die Tone, die niedrige Plastizitätszahlen zeigen, können oft nur zu dicken Drähten, nicht zu dünnen Drähten ausgerollt werden. Das verschiedene Verhalten, ent-

') Bei der Behandlung der Konsistenzlehre. 
weder zu dicken oder ebenfalls zu dünnen Drähten ausgerollt werden zu können, ist jedoch wohl unzweifelhaft eine Folge der verschiedenen Zähigkeit der Tone.

Der Plastizitätsgrad ist somit kein einfacher Begriff. In richtiger Beurteilung der Plastizitätsart muB man sowohl die Plastizitätszahl (die größere oder kleinere Umfassung des Plastizitätsgebietes), wie die Zähigkeit des Tones (die wechselnde Konsistenz innerhalb des Plastizitätsgebietes in Betracht ziehen.

Als Maß der Zähigkeit kann das Bindevermögen (die Menge Sand, welche der Ton binden kann, ohne die Plastizität zu verlieren) dienen, denn das größere oder kleinere Bindevermögen ist sicherlich von der größeren oder kleineren Zähigkeit des Tones abhängig. Richtiger wäre es zwar, die Zähigkeit direkt nach den Methoden der Konsistenzgradbestimmungen zu bestimmen. Das ist jedoch mit Schwierigkeiten verbunden; denn es ist nicht leicht, eine größere Tonprobe gerade von dem Wassergehalte der Ausrollgrenze (wo die Zähigkeit am größten ist) zu bereiten. Das Bindevermögen scheint mir daher den bequemsten Maßstab der Zähigkeit zu liefern.

Ich habe gefunden, daß bei den nordischen Meerestonen die Zähigkeitsziffern mit den Plastizitätszahlen stets steigen und fallen. Bei diesen Tonen sind somit Zähigkeitsbestimmungen überflüssig. Humose Tone bilden aber Ausnahmen und künstliche Tone, aus Kaolinit oder Glimmer bereitet, haben niedrige Zähigkeit bei hohen Plastizitätszahlen erwiesen. Bei humosen Tonen, Kaolinit- und Glimmertonen hat man daher zur richtigen Beurteilung des „Plastizitätsgrades“, der Plastizitätsart, nicht nur die Plastizitätszahlen, sondern ebenfalls die Zähigkeit innerhalb des Plastizitätsgebietes zu bestimmen.

Die Einwirkung verschiedener Einflüsse auf die Plastizitätszahlen.

Daß der Einfluß des Humusgehaltes auf die Eigenschaften der Tone groß ist, ist schon wohlbekannt und geht ebenfalls aus meinem oben angeführten Diagramm hervor. Ein Humusgehalt erhöht die Lage der Plastizitätsgrenzen (d. h. erhöht die Wasseraufnahme, die "Wasserkapazität" der Tone). Die relative Lage der Klebegrenze steigt so stark, daf bei den humosen Tonen die klebende Plastizität ganz verschwindet. Die Plastizitätszahlen sinken aber; der Humusgehalt erniedrigt die Plastizität. 
Eine Sandbeimischung verhält sich fast ebenso. Die Plastizitätszahlen sinken. Die relative Lage der Klebegrenze steigt und die Tone werden nicht klebend.

Die Plastizität der Tone scheint sich bei wechselnder Temperatur nur wenig oder fast gar nicht zu verändern. Höhere Salzgehalte scheinen ebenfalls nicht viel einzuwirken. Ein Ton mit 1 Proz. Salzgehalt zeigte die Plastizitätszahl 17. Ein Sodaboden aus Ungarn zeigte die Plastizitätszahl 25.

\section{Die nähere Feststellung der Lage der Plastizitätsgrenzen.}

Zur Feststellung der Plastizitätszahlen (des Umfanges des Plastizitätsgebietes) muß man die Lage der Plastizitätsgrenzen genau feststellen können. Die Lage dieser Grenzen wird in der folgenden Weise bestimmt.

Die Flie Bgrenze. In einer kleinen Porzellanschale mit rundem Boden und von $10-12 \mathrm{~cm}$ Weite werden etwa $5 \mathrm{~g}$ Tonpulver seitlich in der Schale mit Wasser zu einem Brei ausgerührt. Dem Breie wird allmählich mehr Tonpulver zugesetzt und mit Hilfe eines Nickelspatels damit innig vermischt. Nach jedem Zusatze wird der Tonbrei in der folgenden Weise untersucht. Der Teig wird mit Hilfe des Nickelspatels so ausgeflacht, daß die Höhe der Tonschicht etwas weniger als $1 \mathrm{~cm}$ beträgt. Dann wird der Ton mittels des Nickelspatels in zwei Teile derart geteilt, daß die beiden Hälften die Form und Lage in der Porzellanschale bekommen, wie die erste der folgenden Zeichnungen zeigt. Die Schale wird dann heftig und wiederholt gegen die Innenseite der Hand gestoßen, um die beiden Hälften des Tonbreies zum Zusammenfließen zu bringen.

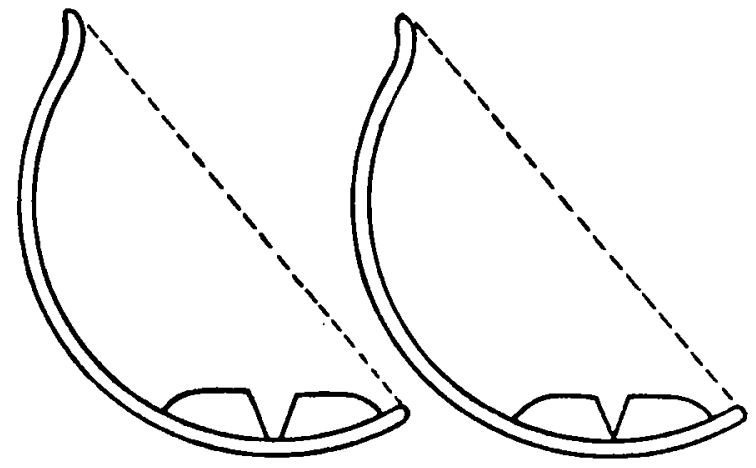

Fig. 3 
Sobald nach erneuten Zusätzen von Tonpulver die beiden Tonhälften in dem untersten Teile der Furche nur ganz unbedeutend, fast gar nicht, zusammenfließen wollen, ist die Grenze erreicht. Die Lage der Grenze wird dann durch Bestimmung des Wassergehaltes des Breies festgestellt. - Diese Grenze ist nur bei den Lehmen scharf. Bei den Tonen ist dieselbe weniger scharf und die Vorschriften der Bestimmung müssen daher genau befolgt werden.

Die A us rollgre nze. Ein Teil des bei den vorigen Bestimmungen erhaltenen Tonteiges, oft vorteilhaft mit etwas Tonpulver gemischt, wird unter den Fingern auf einer Unterlage von Papier zu Drähten ausgerollt. Die Drähte werden wiederholt zusammengelegt und wieder ausgerollt, bis sie bei dem Ausrollversuche zu Bröckchen zergehen. Der Punkt an dem die Drähte zu kürzeren Stückchen zergehen, hat keine Bedeutung. Nur müssen die Stückchen, wenn zusammengelegt, sich fortwährend ausrollen lassen. Es ist aber nicht erlaubt, die Drähte so zu rollen, daß sie sich nicht in der Länge strecken, denn dabei wird die Lage der Grenze leicht überschritten; sondern es müssen sich die Finger bei dem Rollen trennen, damit der Teig gestreckt wird. $\mathrm{Da}$ die Drahtstücke sich nicht länger ausrollen lassen, sondern $\mathrm{zu}$ Bröckchen zergehen, ist die Grenze erreicht. Bei den hochplastischen Tonen werden die Drähte schließlich sehr zäh und gehen nur langsam in Bröckchen über. - Diese Grenze läßt sich mit großer Schärfe bestimmen.

Die Klebegrenze. Man vermische den klebenden Tonteig in der Porzellanschale mit immer mehr Tonmehl, bis der Nickelspatel, durch Ziehen über die Oberfläche des Tonteiges, sich von anhängenden Tonklumpen völlig, wenn auch etwas schwierig, befreien läßt. Die Lage der Grenze wird wie die Lagen der vorigen Grenzen, durch eine Bestimmung des Wassergehaltes des Tonteiges festgestellt.

Sämtliche Wassergehaltsziffern werden nicht in Prozenten, sondern auf 100 Teile der bei $100^{\circ}$ getrockneten Tonprobe berechnet.

\section{Die Klassifikation der Tone nach deren Plastizitätsziffern.}

Die Tone lassen sich nach den oben aufgestellten „Plastizitätsklassen “, d. h. nach deren „Bindevermögen“, einteilen. Die Einteilung nach Plastizitätsklassen muß aber weniger scharf sein, da es nicht leicht ist, stets Sand ganz derselben Art für die Bestimmung des Bindevermögens $z u$ finden. Bequemer und schärfer ist die Einteilung 
der Tone nach den Plastizitätszahlen, die, wie oben gesagt, von 1 bis zu 40 wechseln können.

Die Tone werden dazu vorteilhaft in zwei Hauptklassen geteilt: die Tone, die "klebende Plastizität " zeigen, und die Tone, welche klebende Plastizität nicht zeigen. Die erste Klasse fällt in den meisten Fällen mit den Tonen zusammen, die bei den mechanischen Analysen höhere Gehalte an kolloiden Teilchen als 50 Proz. zeigen. Diese Klasse ist die Klasse der hochplastischen Tone. Die zweite Klasse, deren Tone klebende Plastizität nicht zeigen, fallt dagegen mit den Tonen zusammen, die bei der mechanischen Analyse mehr als 50 Proz. an Feinsand oder Schluff zeigen, den Feins andtonen und den Schlufftonen, welche Tone meistens als "leh. mige Tone" bezeichnet werden. Die genannten beiden Tonklassen sind daher als gute und natürliche Klassen aufzufassen.

Die Methoden zur Bestimmung der Plastizitätsgrenzen und der Klebegrenzen sind sehr einfach, und es geht viel schneller, diese Grenzen zu bestimmen, als eine mechanische Bodenanalyse auszuführen. Die Plastizitäts- und Klebegrenzbestimmungen nach meinen Methoden müssen daher bei den Tonuntersuchungen vorteilhafte Verwendung finden können.

Die Plastizitätslehre der Böden Ist nur ein Teil der Konsistenzlehre. Ueber die weiteren Teile meiner für die Bodenarten aufgestellten Konsistenzlehre hoffe ich in dieser Zeitschrift bald berichten zu können. Daß̧ Plastizität und hohe Konsistenz Eigenschaften sind, die nur gewissen Gruppen von Teilchen kolloider Größe gehören, werde ich ebenfalls bald in dieser Zeitschrift nachweisen. 